\title{
A worked example of Braun and Clarke's approach to reflexive thematic analysis
}

\author{
David Byrne ${ }^{1}$ (D)
}

Accepted: 6 June 2021 / Published online: 26 June 2021

(c) The Author(s) 2021

\begin{abstract}
Since the publication of their inaugural paper on the topic in 2006, Braun and Clarke's approach has arguably become one of the most thoroughly delineated methods of conducting thematic analysis (TA). However, confusion persists as to how to implement this specific approach to TA appropriately. The authors themselves have identified that many researchers who purport to adhere to this approach-and who reference their work as such - fail to adhere fully to the principles of 'reflexive thematic analysis' (RTA). Over the course of numerous publications, Braun and Clarke have elaborated significantly upon the constitution of RTA and attempted to clarify numerous misconceptions that they have found in the literature. This paper will offer a worked example of Braun and Clarke's contemporary approach to reflexive thematic analysis with the aim of helping to dispel some of the confusion regarding the position of RTA among the numerous existing typologies of TA. While the data used in the worked example has been garnered from health and wellbeing education research and was examined to ascertain educators' attitudes regarding such, the example offered of how to implement the RTA would be easily transferable to many other contexts and research topics.
\end{abstract}

Keywords Reflexive · Thematic analysis · Braun · Clarke · Qualitative

\section{Introduction}

Although the lineage of thematic analysis (TA) can be traced back as far as the early twentieth century (Joffe 2012), it has up until recently been a relatively poorly demarcated and poorly understood method of qualitative analysis. Much of the credit for the recent enlightenment and subsequent increase in interest in TA can arguably be afforded to Braun and Clarke's (2006) inaugural publication on the topic of thematic analysis in the field of psychology. These authors have since published several articles and book chapters, as well as their own book, all of which make considerable contributions to further delineating their approach to TA (see, for example, Braun and Clarke 2012, 2013, 2014, 2019, 2020; Braun et al. 2016; Terry et al. 2017). However, on numerous occasions Braun and

David Byrne

david.byrne@mytudublin.ie

1 Technological University Dublin - Blanchardstown Campus, Dublin, Ireland 
Clarke have identified a tendency for scholars to cite their 2006 article, but fail to fully adhere to their contemporary approach to RTA (see Braun and Clarke 2013, 2019, 2020). Commendably, they have acknowledged that their 2006 paper left several aspect of their approach incompletely defined and open to interpretation. Indeed, the term 'reflexive thematic analysis' only recently came about in response to these misconceptions (Braun and Clarke 2019). Much of their subsequent body of literature in this area addresses these issues and attempts to correct some of the misconceptions in the wider literature regarding their approach. Braun and Clarke have repeatedly iterated that researchers who chose to adopt their approach should interrogate their relevant publications beyond their 2006 article and adhere to their contemporary approach (Braun and Clarke 2019, 2020). The purpose of this paper is to contribute to dispelling some of the confusion and misconceptions regarding Braun and Clarke's approach by providing a worked example of their contemporary approach to reflexive thematic analysis. The worked example will be presented in relation to the author's own research, which examined the attitudes of post-primary educators' regarding the promotion of student wellbeing. This paper is intended to be a supplementary resource for any prospective proponents of RTA, but may be of particular interest to scholars conducting attitudinal studies in an educational context. While this paper is aimed at all scholars regardless of research experience, it may be most useful to research students and their supervisors. Ultimately, the provided example of how to implement the six-phase analysis is easily transferable to many contexts and research topics.

\section{What is reflexive thematic analysis?}

Reflexive thematic analysis is an easily accessible and theoretically flexible interpretative approach to qualitative data analysis that facilitates the identification and analysis of patterns or themes in a given data set (Braun and Clarke 2012). RTA sits among a number of varied approaches to conducting thematic analysis. Braun and Clarke have noted that very often, researchers who purport to have adopted RTA have failed to fully delineate their implementation of RTA, of have confused RTA with other approaches to thematic analysis. The over-riding tendency in this regard is for scholars to mislabel their analysis as RTA, or to draw from a number of different approaches to TA, some of which may not be compatible with each other (Braun and Clarke 2012, 2013, 2019; Terry et al. 2017). In an attempt to resolve this confusion, Braun and Clarke have demarcated the position of RTA among the other forms of thematic analysis by differentiating between three principal approaches to TA: (1) coding reliability TA; (2) codebook approaches to TA, and; (3) the reflexive approach to TA (Braun et al. 2019).

Coding reliability approaches, such as those espoused by Boyatzis (1998) and Joffe (2012), accentuate the measurement of accuracy or reliability when coding data, often involving the use of a structured codebook. The researcher would also seek a degree of consensus among multiple coders, which can be measured using Cohen's Kappa (Braun and Clarke 2013). When adopting a coding reliability approach, themes tend to be developed very early in the analytical process. Themes can be hypothesised based on theory prior to data collection, with evidence to support these hypotheses then gathered from the data in the form of codes. Alternatively, themes can be hypothesised following a degree of familiarisation with the data (Terry et al. 2017). Themes are typically understood to constitute 'domain summaries', or "summaries of what participants said 
in relation to a particular topic or data collection question" (Braun et al. 2019, p. 5), and are likely to be discussed as residing within the data in a positivistic sense.

Codebook approaches, such as framework analysis (Smith and Firth 2011) or template analysis (King and Brooks 2017), can be understood to be something of a midpoint between coding reliability approaches and the reflexive approach. Like coding reliability approaches, codebook approaches adopt the use of a structured codebook and share the conceptualisation of themes as domain summaries. However, codebook approaches are more akin to the reflexive approach in terms of the prioritisation of a qualitative philosophy with regard to coding. Proponents of codebook approaches would typically forgo positivistic conceptions of coding reliability, instead recognising the interpretive nature of data coding (Braun et al. 2019).

The reflexive approach to TA highlights the researcher's active role in knowledge production (Braun and Clarke 2019). Codes are understood to represent the researcher's interpretations of patterns of meaning across the dataset. Reflexive thematic analysis is considered a reflection of the researcher's interpretive analysis of the data conducted at the intersection of: (1) the dataset; (2) the theoretical assumptions of the analysis, and; (3) the analytical skills/resources of the researcher (Braun and Clarke 2019). It is fully appreciated-even expected - that no two researchers will intersect this tripartite of criteria in the same way. As such, there should be no expectation that codes or themes interpreted by one researcher may be reproduced by another (although, this is of course possible). Prospective proponents of RTA are discouraged from attempting to provide accounts of 'accurate' or 'reliable' coding, or pursuing consensus among multiple coders or using Cohen's Kappa values. Rather, RTA is about “the researcher's reflective and thoughtful engagement with their data and their reflexive and thoughtful engagement with the analytic process" (Braun and Clarke 2019, p. 594). Multiple coders may, however, be beneficial in a reflexive manner (e.g. to sense-check ideas, or to explore multiple assumptions or interpretations of the data). If analysis does involve more than one researcher, the approach should be collaborative and reflexive, aiming to achieve richer interpretations of meaning, rather than attempting to achieve consensus of meaning. Indeed, in this sense it would be beneficial for proponents of RTA to remain cognisant that qualitative analysis as a whole does not contend to provide a single or 'correct' answer (Braun and Clarke 2013).

The process of coding (and theme development) is flexible and organic, and very often will evolve throughout the analytical process (Braun et al. 2019). Progression through the analysis will tend to facilitate further familiarity with the data, which may in turn result in the interpretation of new patterns of meaning. This is converse to the use of codebooks, which can often predefine themes before coding. Through the reflexive approach, themes are not predefined in order to 'find' codes. Rather, themes are produced by organising codes around a relative core commonality, or 'central organising concept', that the researcher interprets from the data (Braun and Clarke 2019).

In their 2006 paper, Braun and Clarke (2006) originally conceptualised RTA as a paradigmatically flexible analytical method, suitable for use within a wide range of ontological and epistemological considerations. In recent publications, the authors have moved away from this view, instead defining RTA as a purely qualitative approach. This pushes the use RTA into exclusivity under appropriate qualitative paradigms (e.g. constructionism) (Braun and Clarke 2019, 2020). As opposed to other forms of qualitative analysis such as content analysis (Vaismoradi et al. 2013), and even other forms of TA such as Boyatzis' (1998) approach, RTA eschews any positivistic notions of data interpretation. Braun and Clarke (2019) encourage the researcher to embrace reflexivity, subjectivity and creativity as assets 
in knowledge production, where they argue some scholars, such as Boyatzis (1998), may otherwise construe these assets as threats.

\section{A worked example of reflexive thematic analysis}

The data used in the following example is taken from the qualitative phase of a mixed methods study I conducted, which examined mental health in an educational context. This study set out to understand the attitudes and opinions of Irish post-primary educators with regard to the promotion of students' social and emotional wellbeing, with the intention to feed this information back to key governmental and non-governmental stakeholders such as the National Council for Curriculum and Assessment and the Department of Education. The research questions for this study aimed to examine educators' general attitudes toward the promotion of student wellbeing and towards a set of 'wellbeing guidelines' that had recently been introduced in Irish post-primary schools. I also wanted to identify any potential barriers to wellbeing promotion and to solicit educators' opinions as to what might constitute apposite remedial measures in this regard.

The qualitative phase of this study, from which the data for this example is garnered, involved eleven semi-structured interviews, which lasted approximately 25-30 min each. Participants consisted of core-curriculum teachers, wellbeing curriculum teachers, pastoral care team-members and senior management members. Participants were questioned on their attitudes regarding the promotion of student wellbeing, the wellbeing curriculum, the wellbeing guidelines and their perceptions of their own wellbeing. When conducting these interviews, I loosely adhered to an interview agenda to ensure each of these four key topics were addressed. However, discussions were typically guided by what I interpreted to be meaningful to the interviewee, and would often weave in and out of these different topics.

The research questions for this study were addressed within a paradigmatic framework of interpretivism and constructivism. A key principle I adopted for this study was to reflect educators' own accounts of their attitudes, opinions and experiences as faithfully as was possible, while also accounting for the reflexive influence of my own interpretations as the researcher. I felt RTA was highly appropriate in the context of the underlying theoretical and paradigmatic assumptions of my study and would allow me to ensure qualitative data was collected and analysed in a manner that respected and expressed the subjectivity of participants' accounts of their attitudes, while also acknowledging and embracing the reflexive influence of my interpretations as the researcher.

In the next section, I will outline the theoretical assumptions of the RTA conducted in my original study in more detail. It should be noted that outlining these theoretical assumptions is not a task specific to reflexive thematic analysis. Rather, these assumptions should be addressed prior to implementing any form of thematic analysis (Braun and Clarke 2012, 2019, 2020; Braun et al. 2016). The six-phase process for conducting reflexive thematic analysis will then be appropriately detailed and punctuated with examples from my study.

\subsection{Addressing underlying theoretical assumptions}

Across several publications, Braun and Clarke (2012, 2014, 2020) have identified a number of theoretical assumptions that should be addressed when conducting RTA, or indeed any form of thematic analysis. These assumptions are conceptualised as a series of continua as follows: essentialist versus constructionist epistemologies; experiential versus critical 
orientation to data; inductive versus deductive analyses, and; semantic versus latent coding of data. The aim is not just for the researcher to identify where their analysis is situated on each of these continua, but why the analysis is situated as it is and why this conceptualisation is appropriate to answering the research question(s).

\subsubsection{Essentialist versus constructionist epistemologies}

Ontological and epistemological considerations would usually be determined when a study is first being conceptualised. However, these considerations may become salient again when data analysis becomes the research focus, particularly with regard to mixed methods. The purpose of addressing this continuum is to conceptualise theoretically how the researcher understands their data and the way in which the reader should interpret the findings (Braun and Clarke 2013, 2014). By adhering to essentialism, the researcher adopts a unidirectional understanding of the relationship between language and communicated experience, in that it is assumed that language is a simple reflection of our articulated meanings and experiences (Widdicombe and Wooffiitt 1995). The meanings and systems inherent in constructing these meanings are largely uninterrogated, with the interpretive potential of TA largely unutilised (Braun et al. 2016).

Conversely, researchers of a constructionist persuasion would tend to adopt a bidirectional understanding of the language/experience relationship, viewing language as implicit in the social production and reproduction of both meaning and experience (Burr 1995; Schwandt 1998). A constructionist epistemology has particular implications with regard to thematic analysis, namely that in addition to the recurrence of perceptibly important information, meaningfulness is highly influential in the development and interpretation of codes and themes. The criteria for a theme to be considered noteworthy via recurrence is simply that the theme should present repeatedly within the data. However, what is common is not necessarily meaningful or important to the analysis. Braun and Clarke (2012, p. 37) offer this example:

...in researching white-collar workers' experiences of sociality at work, a researcher might interview people about their work environment and start with questions about their typical workday. If most or all reported that they started work at around 9:00 a.m., this would be a pattern in the data, but it would not necessarily be a meaningful or important one.

Furthermore, there may be varying degrees of conviction in respondents' expression when addressing different issues that may facilitate in identifying the salience of a prospective theme. Therefore, meaningfulness can be conceptualised, firstly on the part of the researcher, with regard to the necessity to identify themes that are relevant to answering the research questions, and secondly on the part of the respondent, as the expression of varying degrees of importance with regard to the issues being addressed. By adopting a constructionist epistemology, the researcher acknowledges the importance of recurrence, but appreciates meaning and meaningfulness as the central criteria in the coding process.

In keeping with the qualitative philosophy of RTA, epistemological consideration regarding the example data were constructionist. As such, meaning and experience was 
interpreted to be socially produced and reproduced via an interplay of subjective and intersubjective construction. ${ }^{1}$

\subsubsection{Experiential versus critical orientation}

An experiential orientation to understanding data typically prioritises the examination of how a given phenomenon may be experienced by the participant. This involves investigating the meaning ascribed to the phenomenon by the respondent, as well as the meaningfulness of the phenomenon to the respondent. However, although these thoughts, feelings and experiences are subjectively and inter-subjectively (re)produced, the researcher would cede to the meaning and meaningfulness ascribed by the participant (Braun and Clarke 2014). Adopting an experiential orientation requires an appreciation that the thoughts, feelings and experiences of participants are a reflection of personal states held internally by the participant. Conversely, a critical orientation appreciates and analyses discourse as if it were constitutive, rather than reflective, of respondents' personal states (Braun and Clarke 2014). As such, a critical perspective seeks to interrogate patterns and themes of meaning with a theoretical understanding that language can create, rather than merely reflect, a given social reality (Terry et al. 2017). A critical perspective can examine the mechanisms that inform the construction of systems of meaning, and therefore offer interpretations of meaning further to those explicitly communicated by participants. It is then also possible to examine how the wider social context may facilitate or impugn these systems of meaning (Braun and Clarke 2012). In short, the researcher uses this continuum to clarify their intention to reflect the experience of a social reality (experiential orientation) or examine the constitution of a social reality (critical orientation).

In the present example, an experiential orientation to data interpretation was adopted in order to emphasise meaning and meaningfulness as ascribed by participants. Adopting this approach meant that this analysis did not seek to make claims about the social construction of the research topic (which would more so necessitate a critical perspective), but rather acknowledged the socially constructed nature of the research topic when examining the subjective 'personal states' of participants. An experiential orientation was most appropriate as the aim of the study was to prioritise educators' own accounts of their attitudes, opinions. More importantly, the research questions aimed to examine educators' attitudes regarding their experience of promoting student wellbeing — or the 'meanings made' —and not, for example, the socio-cultural factors that may underlie the development of these attitudes—or the 'meaning making'.

\subsubsection{Inductive versus deductive analysis}

A researcher who adopts a deductive or 'theory-driven' approach may wish to produce codes relative to a pre-specified conceptual framework or codebook. In this case, the analysis would tend to be 'analyst-driven', predicated on the theoretically informed interpretation of the researcher. Conversely, a researcher who adopts an inductive or 'data-driven' approach may wish to produce codes that are solely reflective of the content of the data, free from any pre-conceived theory or conceptual framework. In this case, data are not

\footnotetext{
${ }^{1}$ While the reconceptualisation of RTA as falling within the remit of a purely qualitative paradigm precipitates that the research fall on the constructionist end of this continuum, it is nevertheless good practice to explicate this theoretical position.
} 
coded to fit a pre-existing coding frame, but instead 'open-coded' in order to best represent meaning as communicated by the participants (Braun and Clarke 2013). Data analysed and coded deductively can often provide a less rich description of the overall dataset, instead focusing on providing a detailed analysis of a particular aspect of the dataset interpreted through a particular theoretical lens (Braun and Clarke 2020). Deductive analysis has typically been associated with positivistic/essentialist approaches (e.g. Boyatzis 1998), while inductive analysis tends to be aligned with constructivist approaches (e.g. Frith and Gleeson 2004). That being said, inductive/deductive approaches to analysis are by no means exclusively or intrinsically linked to a particular epistemology.

Coding and analysis rarely fall cleanly into one of these approaches and, more often than not, use a combination of both (Braun and Clarke 2013, 2019, 2020). It is arguably not possible to conduct an exclusively deductive analysis, as an appreciation for the relationship between different items of information in the data set is necessary in order to identify recurring commonalities with regard to a pre-specified theory or conceptual framework. Equally, it is arguably not possible to conduct an exclusively inductive analysis, as the researcher would require some form of criteria to identify whether or not a piece of information may be conducive to addressing the research question(s), and therefore worth coding. When addressing this issue, Braun and Clarke (2012) clarify that one approach does tend to predominate over the other, and that the predominance of the deductive or inductive approach can indicate an overall orientation towards prioritising either researcher/theorybased meaning or respondent/data-based meaning, respectively.

A predominantly inductive approach was adopted in this example, meaning data was open-coded and respondent/data-based meanings were emphasised. A degree of deductive analysis was, however, employed to ensure that the open-coding contributed to producing themes that were meaningful to the research questions, and to ensure that the respondent/ data-based meanings that were emphasised were relevant to the research questions.

\subsubsection{Semantic versus latent coding}

Semantic codes are identified through the explicit or surface meanings of the data. The researcher does not examine beyond what a respondent has said or written. The production of semantic codes can be described as a descriptive analysis of the data, aimed solely at presenting the content of the data as communicated by the respondent. Latent coding goes beyond the descriptive level of the data and attempts to identify hidden meanings or underlying assumptions, ideas, or ideologies that may shape or inform the descriptive or semantic content of the data. When coding is latent, the analysis becomes much more interpretive, requiring a more creative and active role on the part of the researcher. Indeed, Braun and Clarke $(2012,2013,2020)$ have repeatedly presented the argument that codes and themes do not 'emerge' from the data or that they may be residing in the data, waiting to be found. Rather, the researcher plays an active role in interpreting codes and themes, and identifying which are relevant to the research question(s). Analyses that use latent coding can often overlap with aspects of thematic discourse analysis in that the language used by the respondent can be used to interpret deeper levels of meaning and meaningfulness (Braun and Clarke 2006).

In this example, both semantic and latent coding were utilised. No attempt was made to prioritise semantic coding over latent coding or vice-versa. Rather, semantic codes were produced when meaningful semantic information was interpreted, and latent codes were produced when meaningful latent information was interpreted. As such, any item 
of information could be double-coded in accordance with the semantic meaning communicated by the respondent, and the latent meaning interpreted by the researcher (Patton 1990). This was reflective of the underlying theoretical assumptions of the analysis, as the constructive and interpretive epistemology and ontology were addressed by affording due consideration to both the meaning constructed and communicated by the participant and my interpretation of this meaning as the researcher.

\subsection{The six-phase analytical process}

Braun and Clarke $(2012,2013,2014,2020)$ have proposed a six-phase process, which can facilitate the analysis and help the researcher identify and attend to the important aspects of a thematic analysis. In this sense, Braun and Clarke (2012) have identified the six-phase process as an approach to doing TA, as well as learning how to do TA. While the six phases are organised in a logical sequential order, the researcher should be cognisant that the analysis is not a linear process of moving forward through the phases. Rather, the analysis is recursive and iterative, requiring the researcher to move back and forth through the phases as necessary (Braun and Clarke 2020). TA is a time consuming process that evolves as the researcher navigates the different phases. This can lead to new interpretations of the data, which may in turn require further iterations of earlier phases. As such, it is important to appreciate the six-phase process as a set of guidelines, rather than rules, that should be applied in a flexible manner to fit the data and the research question(s) (Braun and Clarke 2013, 2020).

\subsubsection{Phase one: familiarisation with the data}

The 'familiarisation' phase is prevalent in many forms of qualitative analysis. Familiarisation entails the reading and re-reading of the entire dataset in order to become intimately familiar with the data. This is necessary to be able to identify appropriate information that may be relevant to the research question(s). Manual transcription of data can be a very useful activity for the researcher in this regard, and can greatly facilitate a deep immersion into the data. Data should be transcribed orthographically, noting inflections, breaks, pauses, tones, etc. on the part of both the interviewer and the participant (Braun and Clarke 2013). Often times, data may not have been gathered or transcribed by the researcher, in which case, it would be beneficial for the researcher to watch/listen to video or audio recordings to achieve a greater contextual understanding of the data. This phase can be quite time consuming and requires a degree of patience. However, it is important to afford equal consideration across the entire depth and breadth of the dataset, and to avoid the temptation of being selective of what to read, or even 'skipping over' this phase completely (Braun and Clarke 2006).

At this phase, I set about familiarising myself with the data by firstly listening to each interview recording once before transcribing that particular recording. This first playback of each interview recording required 'active listening' and, as such, I did not take any notes at this point. I performed this active-listen in order to develop an understanding of the primary areas addressed in each interview prior to transcription. This also provided me an opportunity, unburdened by tasks such as note taking, to recall gestures and mannerisms that may or may not have been documented in interview notes. I manually transcribed each interview immediately after the active-listen playback. When transcription of all interviews was complete, I read each transcripts numerous times. At this point, I took note of casual 
"Participants heavily emphasise active and practical measures as the most appropriate method to promote student wellbeing".

"Participants often speak of wellbeing in lay terms. It seems they are unaware of any kind of theoretical model of wellbeing. Further, participants do not seem to have intimate knowledge of relevant wellbeing policy or instructional documents".

"There appears to be a discrepancy in the availability of wellbeing-related continuous professional development (CPD). Teachers who deliver the wellbeing curriculum or who fill a pastoral care role (wellbeing educators) have fewer issues attending such CPD. Core curriculum subject teachers (nonwellbeing educators) often receive no such CPD. This may have implications for the whole school approach to wellbeing promotion".

"The core curriculum is prioritised over the wellbeing curriculum".

"Affective dispositions communicated by participants seem to be predominantly: enthusiasm in relation to the value of promoting student wellbeing; frustration at the perceived lack of available training and support, and; resent at the perceived lack of recognition of their historic efforts regarding the promotion of student wellbeing".

Box 1 Example of preliminary notes taken during phase one

observations of initial trends in the data and potentially interesting passages in the transcripts. I also documented my thoughts and feelings regarding both the data and the analytical process (in terms of transparency, it would be beneficial to adhere to this practice throughout the entire analysis). Some preliminary notes made during the early iterations of familiarisation with the data can be seen in Box 1. It will be seen later that some of these notes would go on to inform the interpretation of the finalised thematic framework.

\subsubsection{Phase two: generating initial codes}

Codes are the fundamental building blocks of what will later become themes. The process of coding is undertaken to produce succinct, shorthand descriptive or interpretive labels for pieces of information that may be of relevance to the research question(s). It is recommended that the researcher work systematically through the entire dataset, attending to each data item with equal consideration, and identifying aspects of data items that are interesting and may be informative in developing themes. Codes should be brief, but offer sufficient detail to be able to stand alone and inform of the underlying commonality among constituent data items in relation to the subject of the research (Braun and Clarke 2012; Braun et al. 2016).

A brief excerpt of the preliminary coding process of one participant's interview transcript is presented in Box 2. The preliminary iteration of coding was conducted using the 'comments' function in Microsoft Word (2016). This allowed codes to be noted in the side margin, while also highlighting the area of text assigned to each respective code. This is a relatively straightforward example with no double-codes or overlap in data informing different codes, as new codes begin where previous codes end. The code $\mathrm{C} 5$ offers an exemplar of the provision of sufficient detail to explain what I interpreted from the related data item. A poor example of this code would be to say "the wellbeing guidelines are not 


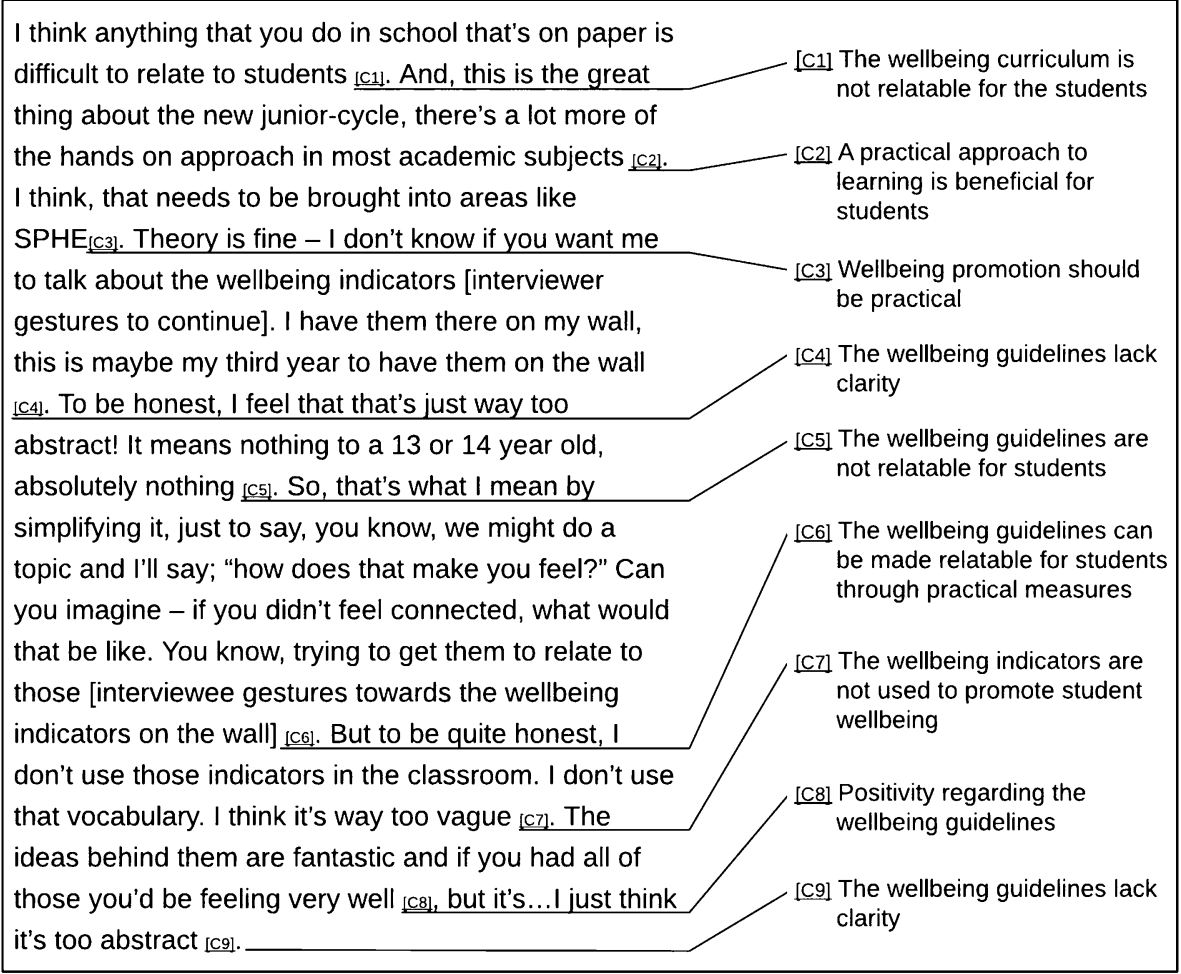

Box 2 Extract of preliminary coding

relatable" or "not relatable for students". Each of these examples lack context. Understanding codes written in this way would be contingent upon knowledge of the underlying data extract. The code $\mathrm{C} 8$ exemplifies this issue. It is unclear if the positivity mentioned relates to the particular participant, their colleagues, or their students. This code was subsequently redefined in later iterations of coding. It can also be seen in this short example that the same code has been produced for both $\mathrm{C} 4$ and $\mathrm{C} 9$. This code was prevalent throughout the entire dataset and would subsequently be informative in the development of a theme.

Any item of data that might be useful in addressing the research question(s) should be coded. Through repeated iterations of coding and further familiarisation, the researcher can identify which codes are conducive to interpreting themes and which can be discarded. I would recommend that the researcher document their progression through iterations of coding to track the evolution of codes and indeed prospective themes. RTA is a recursive process and it is rare that a researcher would follow a linear path through the six phases (Braun and Clarke 2014). It is very common for the researcher to follow a particular train of thought when coding, only to encounter an impasse where several different interpretations of the data come to light. It may be necessary to explore each of these prospective options to identify the most appropriate path to follow. Tracking the evolution of codes will not only aid transparency, but will afford the researcher signposts and waypoints to which they may return should a particular approach to coding prove unfruitful. I tracked the evolution of my coding 


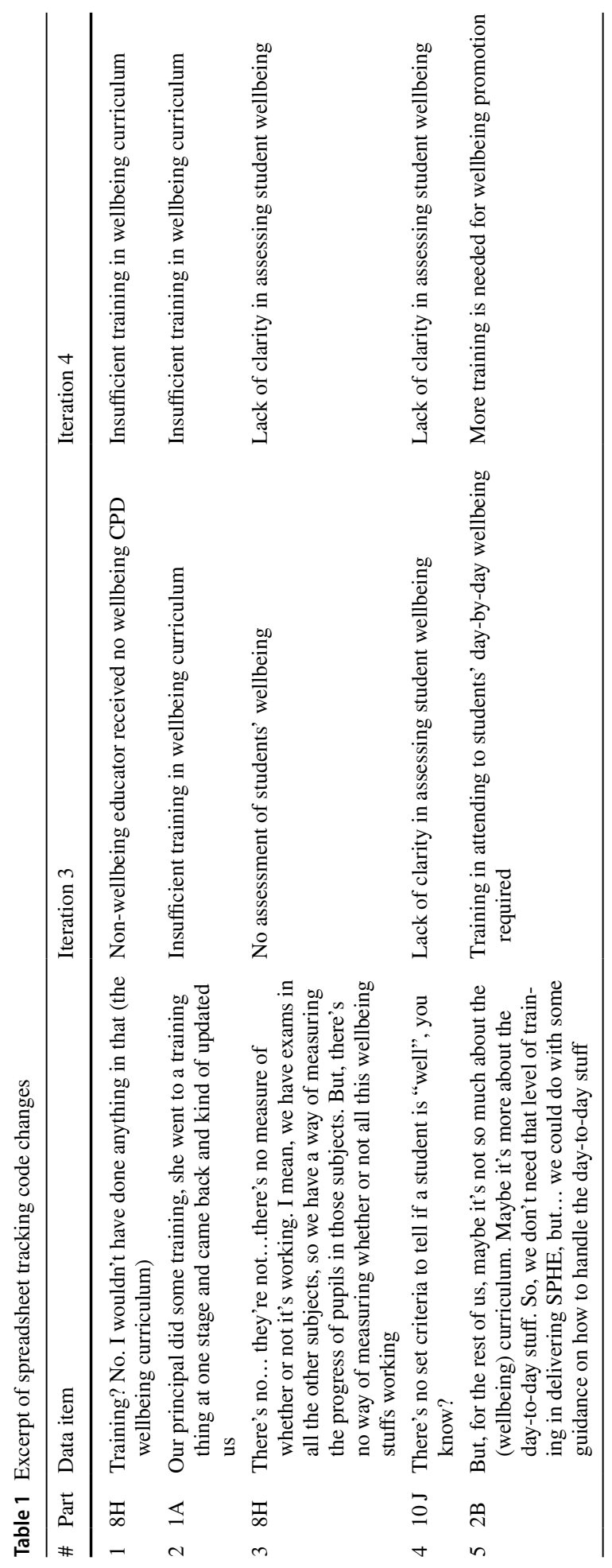


process in a spreadsheet, with data items documented in the first column and iterations of codes in each successive column. I found it useful to highlight which codes were changed in each successive iteration. Table 1 provides an excerpt of a Microsoft Excel (2016) spreadsheet that was established to track iterations of coding and document the overall analytical process. All codes developed during the first iteration of coding were transferred into this spreadsheet along with a label identifying the respective participant. Subsequent iterations of coding were documented in this spreadsheet. The original transcripts were still regularly consulted to assess existing codes and examine for the interpretation of new codes as further familiarity with the data developed. Column one presents a reference number for the data item that was coded, while column two indicates the participant who provided each data item. Column three presents the data item that was coded. Columns four and five indicate the iteration of the coding process to be the third and fourth iteration, respectively. Codes revised between iterations three and four are highlighted.

With regard to data item one, I initially considered that a narrative might develop exploring a potential discrepancy in levels of training received by wellbeing educators and non-wellbeing educators. In early iterations of coding, I adopted a convention of coding training-related information with reference to the wellbeing or non-wellbeing status of the participant. While this discrepancy in levels of training remained evident throughout the dataset, I eventually deemed it unnecessary to pursue interpretation of the data in this way. This coding convention was abandoned at iteration four in favour of the pre-existing generalised code "insufficient training in wellbeing curriculum". With data item three, I realised that the code was descriptive at a semantic level, but not very informative. Upon re-evaluating this data item, I found the pre-existing code "lack of clarity in assessing student wellbeing" to be much more appropriate and representative of what the participant seemed to be communicating. Finally, I realised that the code for data item five was too specific to this particular data item. No other data item shared this code, which would preclude this code (and data item) from consideration when construction themes. I decided that this item would be subsumed under the pre-existing code "more training is needed for wellbeing promotion".

The process of generating codes is non-prescriptive regarding how data is segmented and itemised for coding, and how many codes or what type of codes (semantic or latent) are interpreted from an item of data. The same data item can be coded both semantically and latently if deemed necessary. For example, when discussing how able they felt to attend to their students' wellbeing needs, one participant stated "...if someone's struggling a bit with their schoolwork and it's getting them down a bit, it's common sense that determines what we say to them or how we approach them. And it might help to talk, but I don't know that it has a lasting effect" [2B]. Here, I understood that the participant was explicitly sharing the way in which they address their students' wellbeing concerns, but also that the participant was implying that this commonsense approach might not be sufficient. As such, this data item was coded both semantically as "educators rely on common sense when attending to wellbeing issues", and latently as "common sense inadequate for wellbeing promotion". Both codes were revised later in the analysis. However, this example illustrates the way in which any data item can be coded in multiple ways and for multiple meanings. There is also no upper or lower limit regarding how many codes should be interpreted. What is important is that, when the dataset is fully coded and codes are collated, sufficient depth exists to examine the patterns within the data and the diversity of the positions held by participants. It is, 


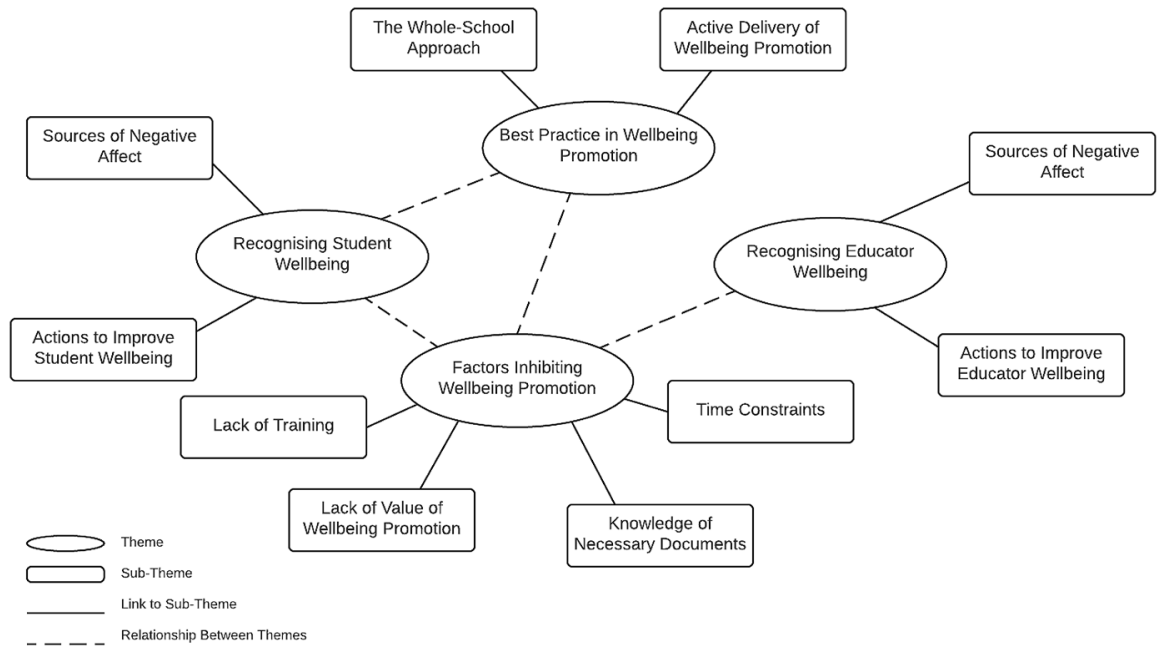

Fig. 1 Initial thematic map indicating four candidate themes

however, necessary to ensure that codes pertain to more than one data item (Braun and Clarke 2012).

\subsubsection{Phase three: generating themes}

This phase begins when all relevant data items have been coded. The focus shifts from the interpretation of individual data items within the dataset, to the interpretation of aggregated meaning and meaningfulness across the dataset. The coded data is reviewed and analysed as to how different codes may be combined according to shared meanings so that they may form themes or sub-themes. This will often involve collapsing multiple codes that share a similar underlying concept or feature of the data into one single code. Equally, one particular code may turn out to be representative of an over-arching narrative within the data and be promoted as a sub-theme or even a theme (Braun and Clarke 2012). It is important to reemphasise that themes do not reside in the data waiting to be found. Rather, the researcher must actively construe the relationship among the different codes and examine how this relationship may inform the narrative of a given theme. Construing the importance or salience of a theme is not contingent upon the number of codes or data items that inform a particular theme. What is important is that the pattern of codes and data items communicates something meaningful that helps answer the research question(s) (Braun and Clarke 2013).

Themes should be distinctive and may even be contradictory to other themes, but should tie together to produce a coherent and lucid picture of the dataset. The researcher must be able and willing to let go of codes or prospective themes that may not fit within the overall analysis. It may be beneficial to construct a miscellaneous theme (or category) to contain all the codes that do not appear to fit in among any prospective themes. This miscellaneous theme may end up becoming a theme in its own right, or may simple be removed from the analysis during a later phase (Braun and Clarke 2012). Much the same as with codes, there is no correct amount of themes. However, with too many themes the analysis may become unwieldy and incoherent, whereas too few themes can result in the analysis failing to explore fully the depth and breadth of the data. At the end of this stage, the researcher 
should be able to produce a thematic map (e.g. a mind map or affinity map) or table that collates codes and data items relative to their respective themes (Braun and Clarke 2012, 2020).

At this point in the analysis, I assembled codes into initial candidate themes. A thematic map of the initial candidate themes can be seen in Fig. 1. The theme "best practice in wellbeing promotion" was clearly definable, with constituent coded data presenting two concurrent narratives. These narratives were constructed as two separate sub-themes, which emphasised the involvement of the entire school staff and the active pursuit of practical measures in promoting student wellbeing, respectively. The theme "recognising student wellbeing" was similarly clear. Again, I interpreted a dichotomy of narratives. However, in this case, the two narratives seemed to be even more synergetic. The two sub-themes for "best practice..." highlighted two independently informative factors in best practice. Here, the sub-themes are much more closely related, with one sub-theme identifying factors that may inhibit the development of student wellbeing, while the second sub-theme discusses factors that may improve student wellbeing. At this early stage in the analysis, I was considering that this sub-theme structure might also be used to delineate the theme "recognising educator wellbeing". Finally, the theme "factors influencing wellbeing promotion" collated coded data items that addressed inhibitive factors with regard to wellbeing promotion. These factors were conceptualised as four separate sub-themes reflecting a lack of training, a lack of time, a lack of appropriate value for wellbeing promotion, and a lack of knowledge of supporting wellbeing-related documents. While it was useful to bring all of this information together under one theme, even at this early stage it was evident that this particular theme was very dense and unwieldy, and would likely require further revision.

\subsubsection{Phase four: reviewing potential themes}

This phase requires the researcher to conduct a recursive review of the candidate themes in relation to the coded data items and the entire dataset (Braun and Clarke 2012, 2020). At this phase, it is not uncommon to find that some candidate themes may not function well as meaningful interpretations of the data, or may not provide information that addresses the research question(s). It may also come to light that some of the constituent codes and/ or data items that inform these themes may be incongruent and require revision. Braun and Clarke (2012, p. 65) proposed a series of key questions that the researcher should address when reviewing potential themes. They are:

- Is this a theme (it could be just a code)?

- If it is a theme, what is the quality of this theme (does it tell me something useful about the data set and my research question)?

- What are the boundaries of this theme (what does it include and exclude)?

- Are there enough (meaningful) data to support this theme (is the theme thin or thick)?

- Are the data too diverse and wide ranging (does the theme lack coherence)?

The analysis conducted at this phase involves two levels of review. Level one is a review of the relationships among the data items and codes that inform each theme and sub-theme. If the items/codes form a coherent pattern, it can be assumed that the candidate theme/ sub-theme makes a logical argument and may contribute to the overall narrative of the data. At level two, the candidate themes are reviewed in relation to the data set. Themes are assessed as to how well they provide the most apt interpretation of the data in relation to 


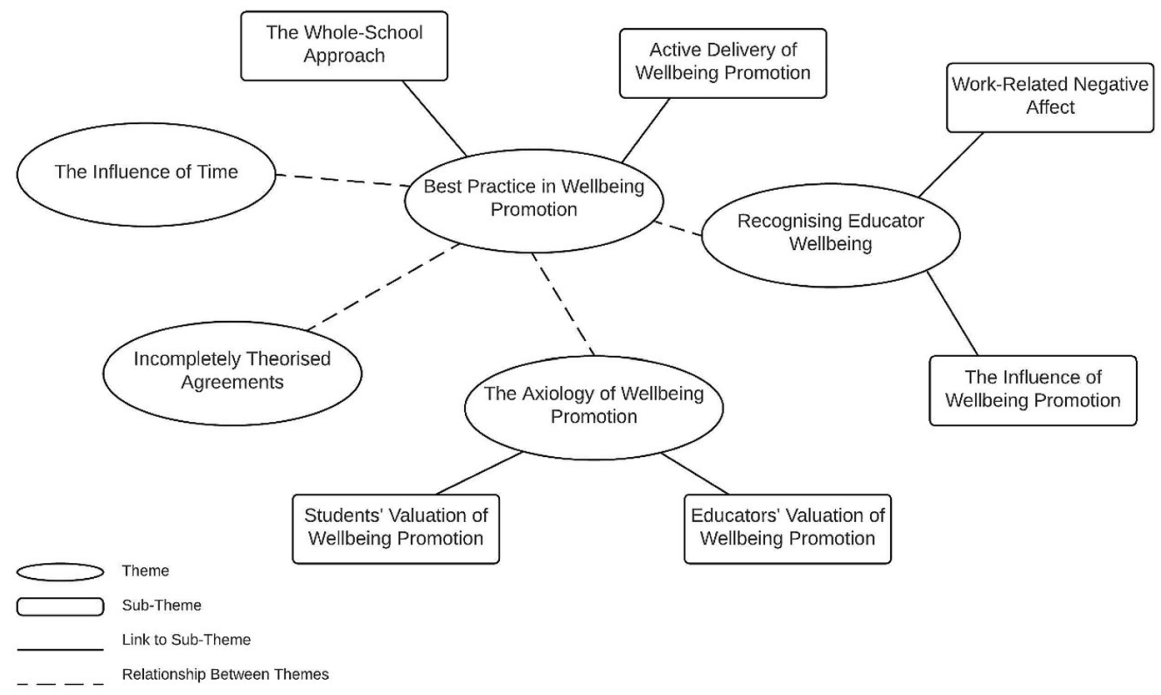

Fig. 2 Finalised thematic map demonstrating five themes

the research question(s). Braun and Clarke have proposed that, when addressing these key questions, it may be useful to observe Patton's (1990) 'dual criteria for judging categories' (i.e. internal homogeneity and external heterogeneity). The aim of Patton's dual criteria would be to observe internal homogeneity within themes at the level one review, while observing external heterogeneity among themes at the level two review. Essentially, these two levels of review function to demonstrate that items and codes are appropriate to inform a theme, and that a theme is appropriate to inform the interpretation of the dataset (Braun and Clarke 2006). The outcome of this dual-level review is often that some sub-themes or themes may need to be restructured by adding or removing codes, or indeed adding or removing themes/sub-themes. The finalised thematic framework that resulted from the review of the candidate themes can be seen in Fig. 2.

During the level one review, inspection of the prospective sub-theme "sources of negative affect" in relation to the theme "recognising educator wellbeing" resulted in a new interpretation of the constituent coded data items. Participants communicated numerous pre-existing work-related factors that they felt had a negative impact upon their wellbeing. However, it was also evident that participants felt the introduction of the new wellbeing curriculum and the newly mandated task of formally attending to student wellbeing had compounded these pre-existing issues. While pre-existing issues and wellbeing-related issues were both informative of educators' negative affect, the new interpretation of this data informed the realisation of two concurrent narratives, with wellbeing-related issues being a compounding factor in relation to pre-existing issues. This resulted in the "sources of negative affect" sub-theme being split into two new sub-themes; "work-related negative affect" and "the influence of wellbeing promotion". The "actions to improve educator wellbeing" sub-theme was folded into these sub-themes, with remedial measures for each issue being discussed in respective sub-themes.

During the level two review, my concerns regarding the theme "factors inhibiting wellbeing promotion" were addressed. With regard to Braun and Clarke's key questions, it was quite difficult to identify the boundaries of this theme. It was also particularly dense (or too 
thick) and somewhat incoherent. At this point, I concluded that this theme did not constitute an appropriate representation of the data. Earlier phases of the analysis were reiterated and new interpretations of the data were developed. This candidate theme was subsequently broken down into three separate themes. While the sub-themes of this candidate theme were, to a degree, informative in the development of the new themes, the way in which the constituent data was understood was fundamentally reconceptualised. The new theme, entitled "the influence of time", moves past merely describing time constraints as an inhibitive factor in wellbeing promotion. A more thorough account of the bi-directional nature of time constraints was realised, which acknowledged that previously existing time constraints affected wellbeing promotion, while wellbeing promotion compounded previously existing time constraints. This added an analysis of the way in which the introduction of wellbeing promotion also produced time constraints in relation to core curricular activities.

The candidate sub-themes "lack of training" and "knowledge of necessary documents" were re-evaluated and considered to be topical rather than thematic aspects of the data. Upon further inspection, I felt that the constituent coded data items of these two sub-themes were informative of a single narrative of participants attending to their students' wellbeing in an atheoretical manner. As such, these two candidate sub-themes were folded into each other to produce the theme "incompletely theorised agreements". Finally, the level two review led me to the conclusion that the full potential of the data that informed the candidate sub-theme "lack of value of wellbeing promotion" was not realised. I found that a much richer understanding of this data was possible, which was obscured by the initial, relatively simplistic, descriptive account offered. An important distinction was made, in that participants held differing perceptions of the value attributed to wellbeing promotion by educators and by students. Further, I realised that educators' perceptions of wellbeing promotion were not necessarily negative and should not be exclusively presented as an inhibitive factor in wellbeing promotion. A new theme, named "the axiology of wellbeing" and informed by the sub-themes "students' valuation of wellbeing promotion" and "educators' valuation of wellbeing promotion", was developed to delineate this multifaceted understanding of participants' accounts of the value of wellbeing promotion.

It is quite typical at this phase that codes, as well as themes, may be revised or removed to facilitate the most meaningful interpretation of the data. As such, it may be necessary to reiterate some of the activities undertaken during phases two and three of the analysis. It may be necessary to recode some data items, collapse some codes into one, remove some codes, or promote some codes as sub-themes or themes. For example, when re-examining the data items that informed the narrative of the value ascribed to wellbeing promotion, I observed that participants offered very different perceptions of the value ascribed by educators and by students. To pursue this line of analysis, numerous codes were reconceptualised to reflect the two different perspectives. Codes such as "positivity regarding the wellbeing curriculum" were split into the more specified codes "student positivity regarding the wellbeing curriculum" and "educator positivity regarding the wellbeing curriculum". Amending codes in this way ultimately contributed to the reinterpretation of the data and the development of the finalised thematic map.

As with all other phases, it is very important to track and document all of these changes. With regard to some of the more significant changes (removing a theme, for example), I would recommend making notes on why it might be necessary to take this action. The aim of this phase is to produce a revised thematic map or table that captures the most important elements of the data in relation to the research question(s). 


\subsubsection{Phase five: defining and naming theme}

At this phase, the researcher is tasked with presenting a detailed analysis of the thematic framework. Each individual theme and sub-theme is to be expressed in relation to both the dataset and the research question(s). As per Patton's (1990) dual criteria, each theme should provide a coherent and internally consistent account of the data that cannot be told by the other themes. However, all themes should come together to create a lucid narrative that is consistent with the content of the dataset and informative in relation to the research question(s). The names of the themes are also subject to a final revision (if necessary) at this point.

Defining themes requires a deep analysis of the underlying data items. There will likely be many data items underlying each theme. It is at this point that the researcher is required to identify which data items to use as extracts when writing up the results of the analysis. The chosen extracts should provide a vivid and compelling account of the arguments being made by a respective theme. Multiple extracts should be used from the entire pool of data items that inform a theme in order to convey the diversity of expressions of meaning across these data items, and to demonstrate the cohesion of the theme's constituent data items. Furthermore, each of the reported data extracts should be subject to a deep analysis, going beyond merely reporting what a participant may have said. Each extract should be interpreted in relation to its constitutive theme, as well as the broader context of the research question(s), creating an analytic narrative that informs the reader what is interesting about this extract and why (Braun and Clarke 2012).

Data extracts can be presented either illustratively, providing a surface-level description of what participants said, or analytically, interrogating what has been interpreted to be important about what participants said and contextualising this interpretation in relation to the available literature. If the researcher were aiming to produce a more illustrative write-up of the analysis, relating the results to the available literature would tend to be held until the 'discussion' section of the report. If the researcher were aiming to produce an analytical write-up, extracts would tend to be contextualised in relation to the literature as and when they are reported in the 'results' section (Braun and Clarke 2013; Terry et al. 2017). While an illustrative write-up of RTA results is completely acceptable, the researcher should remain cognisant that the narrative of the write-up should communicate the complexities of the data, while remaining "embedded in the scholarly field" (Braun and Clarke 2012, p. 69). RTA is an interpretive approach to analysis and, as such, the overall report should go beyond describing the data, providing theoretically informed arguments as to how the data addresses the research question(s). To this end, a relatively straightforward test can reveal a researcher's potential proclivity towards one particular reporting convention: If an extract can be removed and the write-up still makes sense, the reporting style is illustrative; if an extract is removed and the write-up no longer makes sense, the reporting style is analytical (Terry et al. 2017).

The example in Box 3 contains a brief excerpt from the sub-theme "the whole-school approach", which demonstrates the way in which a data extract may be reported in an illustrative manner. Here, the narrative discussed the necessity of having an 'appropriate educator' deliver the different aspects of the wellbeing curriculum. One participant provided a particularly useful real-world example of the potential negative implications of having 'the wrong person' for this job in relation to physical education (one of the aspects of the wellbeing curriculum). This data extract very much informed the 
"I remember a P.E. teacher who used to be in the army! [laughs] So, he'd have the poor kids run ragged! [laughs]. So that was good in one sense, you know, it whipped them into shape. But...the emphasis was on fitness and not wellness. There were lots of drills, and exercises, and laps of the field! But...how hard they were pushed could upset some pupils. You know, you'd hear them complain about how hard it was. And he'd - that teacher would be complaining about the amount of pupils coming in with notes (from parents) saying they can't do P.E. today, and you're thinking 'are you surprised!?' [laughs] So, you definitely need the right person for the job! [P11]".

This participant identified that, even when an educator is highly trained in an area relative to the wellbeing curriculum, they still may not have a personality appropriate for attending to the wellbeing of young people. The physical educators' prioritisation of physical fitness over holistic wellness was likely influenced by a military background, and evidently had a negative effect upon the overall wellbeing of many students. This negative effect was so pervasive that it ultimately resulted in subject refusal among some students. In this case, the physical educator seemed to be unaware of the inherent issue as communicated by the respondent in that they were unable to deliver a fitness/wellness regime at an appropriate level for many of the students.

Box 3 Example of data extract reported illustratively

narrative and illustrated participants' arguments regarding the importance of choosing an appropriate educator for the job.

In Box 4, an example is offered of how a data extract may be reported in an analytical manner. This excerpt is also taken from the sub-theme "the whole-school approach", and also informs the 'appropriate educator for the job' narrative. Here, however, sufficient evidence has already been established to illustrate the perspectives of the participants. The report turns to a deeper analysis of what has been said and how it has been said. Specifically, the way in which participants seemed to construe an 'appropriate educator' was examined and related to existing literature. The analytical interpretation of this data extract (and others) proposes interesting implications regarding the way in which participants constructed their schema of an 'appropriate educator'.

The names of themes are also subject to a final review (if necessary) at this point. Naming themes may seem trivial and might subsequently receive less attention than it actually requires. However, naming themes is a very important task. Theme names are the first indication to the reader of what has been captured from the data. Names should be concise, informative, and memorable. The overriding tendency may be to create names that are descriptors of the theme. Braun and Clarke (2013, 2014, 2020) encourage creativity and advocate the use of catchy names that may more immediately capture the attention of the reader, while also communicating an important aspect of the theme. To this end, they 
"School management needs to be really careful about who they pick to teach SPHE. So, this is how it works. 'A P.E. teacher has 24 lessons of P.E. He needs another five lessons. I'll throw him into SPHE'. Now, that P.E. teacher might be the last person in the world who should be delivering it because, number one, he (hypothetically) doesn't see any importance in it. Number two, he hasn't the personality, you know, to deliver the stuff around sex, around drugs and alcohol. People shy away from that. They won't have hard conversations with youngsters about that, or anything that's challenging. And that's the sort of people you should not be letting near SPHE [P3].

Discussions around the topic of choosing an appropriate educator to deliver the wellbeing curriculum tended to be informed by negative connotations. As is illustrated in the extract above when the participant declares “...he hasn't the personality..." and "...they won't have hard conversations...", respondents often identified the criteria for what an appropriate SPHE educator is by identifying what an appropriate SPHE educator is not. Particularly at the beginning of discussions regarding this topic, participants were observed to engage in perceived value dissimilarity (Struch \& Schwartz 1989), identifying undesirable criteria and establishing the identity of an appropriate SPHE educator as antithetical to these criteria. Ascribing meaning or value in this way has been strongly associated with negative perceptions of the 'other' group (Struch \& Schwartz 1989; Sirin McCreary \& Mahalik, 2004), in this case the inappropriate educator.

Box 4 Example of data extract reportedanalytically

suggest that it may be useful to examine data items for a short extract that could be used to punctuate the theme name.

\subsubsection{Phase six: producing the report}

The separation between phases five and six can often be blurry. Further, this 'final' phase would rarely only occur at the end of the analysis. As opposed to practices typical of quantitative research that would see the researcher conduct and then write up the analysis, the write-up of qualitative research is very much interwoven into the entire process of the analysis (Braun and Clarke 2012). Again, as with previous phases, this will likely require a recursive approach to report writing. As codes and themes change and evolve over the course of the analysis, so too can the write-up. Changes should be well documented by this phase and reflected in informal notes and memos, as well as a research journal that should be kept over the entire course of the research. Phase six then, can be seen as the completion and final inspection of the report that the researcher would most likely have begun writing before even undertaking their thematic analysis (e.g. a journal article or thesis/dissertation). 
A useful task to address at this point would be to establish the order in which themes are reported. Themes should connect in a logical and meaningful manner, building a cogent narrative of the data. Where relevant, themes should build upon previously reported themes, while remaining internally consistent and capable of communicating their own individual narrative if isolated from other themes (Braun and Clarke 2012). I reported the theme "best practice in wellbeing promotion" first, as I felt it established the positivity that seemed to underlie the accounts provided by all of my participants. This theme was also strongly influence by semantic codes, with participants being very capable of describing what they felt would constitute 'best practice'. I saw this as an easily digestible first theme to ease the reader into the wider analysis. It made sense to report "the axiology of wellbeing promotion" next. This theme introduced the reality that, despite an underlying degree of positivity, participants did indeed have numerous concerns regarding wellbeing promotion, and that participants' attitudes were generally positive with a significant 'but'. This theme provided good sign-posting for the next two themes that would be reported, which were "the influence of time" and "incompletely theorised agreements", respectively. I reported "the influence of time" first, as this theme established how time constraints could negatively affect educator training, contributing to a context in which educators were inadvertently pushed towards adopting incompletely theorised agreements when promoting student wellbeing. The last theme to be reported was "recognising educator wellbeing". As the purpose of the analysis was to ascertain the attitudes of educators regarding wellbeing promotion, it felt appropriate to offer the closing commentary of the analysis to educators' accounts of their own wellbeing. This became particularly pertinent when the sub-themes were revised to reflect the influence of pre-existing work-related issues and the subsequent influence of wellbeing promotion.

An issue proponents of RTA may realise when writing up their analysis is the potential for incongruence between traditional conventions for report writing and the appropriate style for reporting RTA-particularly when adopting an analytical approach to reporting on data. The document structure for academic journal articles and Masters or $\mathrm{PhD}$ theses typically subscribe to the convention of reporting results of analyses in a 'results' section and then synthesising and contextualising the results of analyses in a 'discussion' section. Conversely, Braun and Clarke recommend synthesising and contextualising data as and when they are reported in the 'results' section (Braun and Clarke 2013; Terry et al. 2017). This is a significant departure from the traditional reporting convention, which researchers-particularly post-graduate studentsmay find difficult to reconcile. While Braun and Clarke do not explicitly address this potential issue, it is implicitly evident that they would advocate that researchers prioritise the appropriate reporting style for RTA and not cede to the traditional reporting convention.

\section{Conclusion}

Although Braun and Clarke are widely published on the topic of reflexive thematic analysis, confusion persists in the wider literature regarding the appropriate implementation of this approach. The aim of this paper has been to contribute to dispelling some of this confusion by provide a worked example of Braun and Clarke's contemporary approach to reflexive thematic analysis. To this end, this paper provided instruction in how to address 
the theoretical underpinnings of RTA by operationalising the theoretical assumptions of the example data in relation to the study from which the data was taken. Clear instruction was also provided in how to conduct a reflexive thematic analysis. This was achieved by providing a detailed step-by-step guide to Braun and Clarke's six-phase process, and by providing numerous examples of the implementation of each phase based on my own research. Braun and Clarke have made (and continue to make) an extremely valuable contribution to the discourse regarding qualitative analysis. I strongly recommended that any prospective proponents of RTA who may read this paper thoroughly examine Braun and Clarke's full body of literature in this area, and aim to achieve an understanding of RTA's nuanced position among the numerous different approaches to thematic analysis.

Funding Open Access funding provided by the IReL Consortium. This study was funded by Technological University Dublin Research Scholarship.

\section{Declarations}

Conflict of interest The author declares that he/she has no conflict of interest.

Open Access This article is licensed under a Creative Commons Attribution 4.0 International License, which permits use, sharing, adaptation, distribution and reproduction in any medium or format, as long as you give appropriate credit to the original author(s) and the source, provide a link to the Creative Commons licence, and indicate if changes were made. The images or other third party material in this article are included in the article's Creative Commons licence, unless indicated otherwise in a credit line to the material. If material is not included in the article's Creative Commons licence and your intended use is not permitted by statutory regulation or exceeds the permitted use, you will need to obtain permission directly from the copyright holder. To view a copy of this licence, visit http://creativecommons.org/licenses/by/4.0/.

\section{References}

Boyatzis, R.E.: Transforming Qualitative Information: Thematic Analysis and Code Development. Sage Publications, Thousand Oaks (1998)

Braun, V., Clarke, V.: Using thematic analysis in psychology. Qual. Res. Psychol. 3(2), 77-101 (2006). https://doi.org/10.1191/1478088706qp063oa

Braun, V., Clarke, V.: Thematic analysis. In: Cooper, H., Camic, P.M., Long, D.L., Panter, A.T., Rindskopf, D., Sher, K.J. (eds.) APA Handbook of Research Methods in Psychology, Research Designs, vol. 2, pp. 57-71. American Psychological Association, Washington (2012)

Braun, V., Clarke, V.: Successful Qualitative Research: A Practical Guide for Beginners. Sage Publications, Thousand Oaks (2013)

Braun, V., Clarke, V.: Thematic analysis. In: Teo, T. (ed.) Encyclopedia of Critical Psychology, pp. 19471952. Springer, New York (2014)

Braun, V., Clarke, V.: Reflecting on reflexive thematic analysis. Qual. Res. Sport Exerc. Health 11(4), 589597 (2019). https://doi.org/10.1080/2159676X.2019.1628806

Braun, V., Clarke, V.: One size fits all? What counts as quality practice in (reflexive) thematic analysis? Qual. Res. Psychol. (2020). https://doi.org/10.1080/14780887.2020.1769238

Braun, V., Clarke, V., Weate, P.: Using Thematic Analysis in sport and exercise research. In: Smith, B., Sparkes, A.C. (eds.) Routledge Handbook of Qualitative Research in Sport and Exercise, pp. 191-205. Routledge, London (2016)

Braun, V., Clarke, V., Terry, G., Hayfield, N.: Thematic analysis. In: Liamputtong, P. (ed.) Handbook of Research Methods in Health and Social Sciences, pp. 843-860. Springer, Singapore (2018)

Braun, V., Clarke, V., Hayfield, N., Terry, G.: Answers to frequently asked questions about thematic analysis (2019). Retrieved from https://cdn.auckland.ac.nz/assets/psych/about/our-research/documents/Answe rs\%20to\%20frequently\%20asked\%20questions\%20about\%20thematic\%20analysis\%20April\%202019. pdf 
Burr, V.: An Introduction to Social Constructionism. Routledge, London, UK (1995)

Clarke, V., Braun, V.: Thematic Analysis. In: Lyons, E., Coyle, A. (eds.) Analysing Qualitative Data in Psychology, 2nd edn., pp. 84-103. Sage Publications, London (2016)

Frith, H., Gleeson, K.: Clothing and embodiment: men managing body image and appearance. Psychol. Men Mascul. 5(1), 40-48 (2004). https://doi.org/10.1037/1524-9220.5.1.40

Joffe, H.: Thematic analysis. In: Harper, D., Thompson, A.R. (eds.) Qualitative Research Methods in Mental Health and Psychotherapy: A Guide for Students and Practitioners, pp. 209-223. Wiley, Chichester (2012)

King, N., Brooks, J.M.: Template analysis for business and management students. Sage Publications, London, UK (2017)

Patton, M.Q.: Qualitative Evaluation and Research Methods, 2nd edn. Sage Publications, Thousand Oaks (1990)

Schwandt, T.A.: Constructivist, interpretivist approaches to human inquiry. In: Denzin, N.K., Lincoln, Y.S. (eds.) The Landscape of Qualitative Research: Theories and Issues, pp. 221-259. Sage Publications, Thousand Oaks (1998)

Smith, J., Firth, J.: Qualitative data analysis: The framework approach. Nurse Res. 18(2), 52-62 (2011). https://doi.org/10.7748/nr2011.01.18.2.52.c8284

Terry, G., Hayfield, N., Braun, V., Clarke, V.: Thematic analysis. In: Willig, C., Rogers, W.S. (eds.) The SAGE Handbook of Qualitative Research in Psychology, pp. 17-37. Sage Publications, London (2017)

Vaismoradi, M., Turunen, H., Bondas, T.: Content analysis and thematic analysis: implications for conducting a qualitative descriptive study. Nurs. Health Sci. 15(3), 398-405 (2013). https://doi.org/10.1111/ nhs. 12048

Widdicombe, S., Wooffitt, R.: The Language of Youth Subcultures: Social Identity in Action. Harvester, Hemel Hempstead (1995)

Publisher's Note Springer Nature remains neutral with regard to jurisdictional claims in published maps and institutional affiliations. 